\title{
Neuronal Migration Disorder
}

National Cancer Institute

\section{Source}

National Cancer Institute. Neuronal Migration Disorder. NCI Thesaurus. Code C98990.

A diverse group of congenital brain developmental disorders characterized by defects in neuronal migration in the brain during early fetal development. The neuronal migration defects result in brain abnormalities that are usually manifested with mental retardation and epilepsy. 